\title{
Genetic Algorithm's Application for Optimization of PID Parameters in Dynamic Positioning Vessel
}

\author{
Liu gai ye ; Hou Yuan-bin ; Luo yan ; Li dong \\ School of Electric and Control Engineering, Xi'an University of Science \\ and Technology, Xi' an 710054,China
}

\begin{abstract}
Genetic algorithm as intelligent optimization, There are important applications in many field, The genetic algorithm is applied to the tuning of PID parameters in the dynamic positioning of ship,First,In this paper, a model of three degree of freedom model of ship motion and the ocean environment disturbance model of wind, wave and current are established,Secondly,PID control in dynamic positioning system is,Difficulties in debugging PID parameters, This paper focuses on the use of genetic algorithm to optimize the PID parameters, To achieve the purpose of optimizing the PID,Last,The optimization algorithm is simulated,Through the analysis of simulation results, Come to conclusion, In ship dynamic positioning, When using PID controller, The genetic algorithm can be used to find the optimized PID parameter group.
\end{abstract}

\section{1 introduction}

With the development of the ocean and the expansion of human activities, Marine resources have become a strategic resource of fierce competition among countries. Humans of ocean exploration activities will need to ship and platform structures locate a specific location in the ocean, and traditional way of anchorage due to factors such as water depth, working time, precision requirements for the restrictions cannot meet the requirements of relevant offshore operation. With the development of electronic computer and automatic control theory, the ship control technology of dynamic positioning is generated. The so-called dynamic positioning refers to the ability of the ship's own thrusters to realize its position or preset track in the complex Marine environment automatically.

The control algorithm of ship dynamic positioning is generally based on the classic PID control, PID control has many advantages and has a strong robustness. However, the parameters of the PID control is currently rely mainly on pool test setting, when a multi-channel PID control, there will be a lot of PID parameters, setting the PID parameter together only depend on the test will cost a lot of time and energy. Aiming at this problem, this paper proposes a method of PID control parameter setting based on genetic algorithm, and the optimization of PID parameters in dynamic positioning control is optimized by using genetic algorithm.

\section{Ship dynamic positioning model}

\section{1 kinematics model of ship}

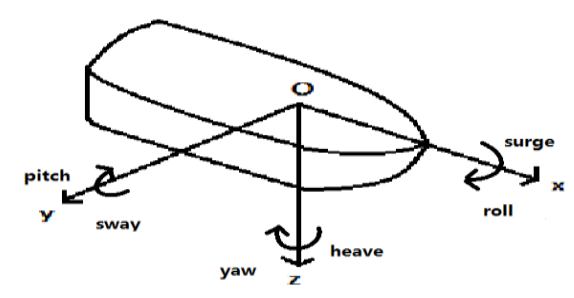

Fig. 1. schematic diagram of ship motion six degrees of freedom

The ship kinematics includes linear motion and angular motion model. According to the literature [1], the ship motion six degrees of freedom can be directly written:

$$
\dot{\eta}=J(\eta) v
$$

It is equivalent to:

$$
\left[\begin{array}{c}
P^{n} \\
\Theta
\end{array}\right]=\left[\begin{array}{cc}
R_{b}^{n}(\Theta) & 0_{3 \times 3} \\
0_{3 \times 3} & T_{\Theta}(\Theta)
\end{array}\right]\left[\begin{array}{c}
U_{o}^{b} \\
\Omega_{n b}^{b}
\end{array}\right]
$$

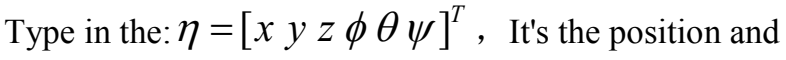
the attitude vector; $v=[u v w p q r]^{T}$, It's the velocity and the angular velocity vector; $J(\eta)$ It's the jacobian transformation matrix.

\section{2 ship dynamics model}

Ship dynamics is the study of ship movement position and attitude change when hull is affected by 
force and torque. According to the literature [1], the ship dynamics model can be directly written:

$$
M \dot{v}+C(v) v+D(v) v+g(\eta)=\tau+g_{o}+w
$$

On the right side of the equation are the various torque items which are applied to the ship, including the control moment and the interference moment.

In order to simplify the problem, this paper studies the ship motion at a lower speed, which can be assumed that the damping is only linear damping ${ }^{\mathbf{2} \mathbf{I}}$, namely

$$
D(v)=D_{L}+D_{N L} \approx D_{L}
$$

$D_{L} 、 D_{N L}$ ( v ) It represents the damping matrix, $C(v) v$ The quadratic velocity is negligible, The quadratic velocity is negligible,

$$
C(v) \approx 0
$$

In this paper, the six-degree-of-freedom model is simplified to three degrees of freedom, swinging and rolling. Its simplified model is:

$$
\dot{\eta}=R(\psi) v
$$

$$
M v+D(v) v=\tau+w
$$

\section{Genetic algorithm for optimization of PID parameters of DP ship and simulation}

\section{1 the PID control}

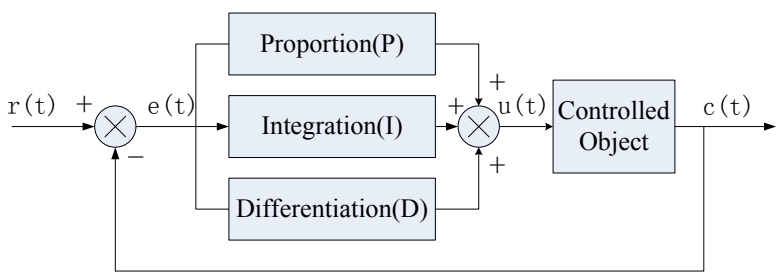

Fig. 2. PID control flow chart The control rules of PID control are expressed as:

$u(t)=K_{p}\left[e(t)+\frac{1}{T_{I}} \int_{0}^{t} e(t) d t+\frac{T_{D} d e(t)}{d t}\right]$

Type, $K_{p}$ Proportional gain, $T_{I}$ Is the integral time constant, $T_{D}$ For the differential time constant, $e(t)$ For the deviation, $u(t)$ To control the amount of 。

PID The functions of each link can be summarized as follows:

(1) proportion : proportional amplification deviation signal. Once the deviation is generated, the controller generates corresponding control to reduce the deviation.
(2) integration : it is mainly to eliminate the static difference of the system and improve the degree of the system. The larger the integral time constant, the weaker the integral action, and the stronger the other.

(3) differential link: the change trend of the deviation is reflected, and the early correction signal can be introduced in the system in advance, so as to speed up the response speed of the system, reduce the shock, and reduce the adjustment time.

\section{2 genetic algorithm}

Genetic Algorithm [ 3 1 (based Algorithm) study began in the late $1960 \mathrm{~s}$ to the early $70 \mathrm{~s}$, mainly by the American professor John Holland and his colleagues at the university of Michigan, the students formed a relatively complete theory and method.

The genetic algorithm begins with a population of possible potential solutions for the problem, and a population is composed of a certain number of individuals that are encoded by genes. Original population carried out in accordance with the fitness of the selection, each subsequent generation evolution produces better approximate solution, in each generation, according to the problems of individual fitness (fitness) size selection (selection) and by means of genetic crosses genetic operators (crossover and mutation (mutation), on behalf of the new solution set of the population. The optimal individual in the last population is decoding, which can be the approximate optimal solution for the problem. The operation process of genetic algorithm can be explained by the following image:

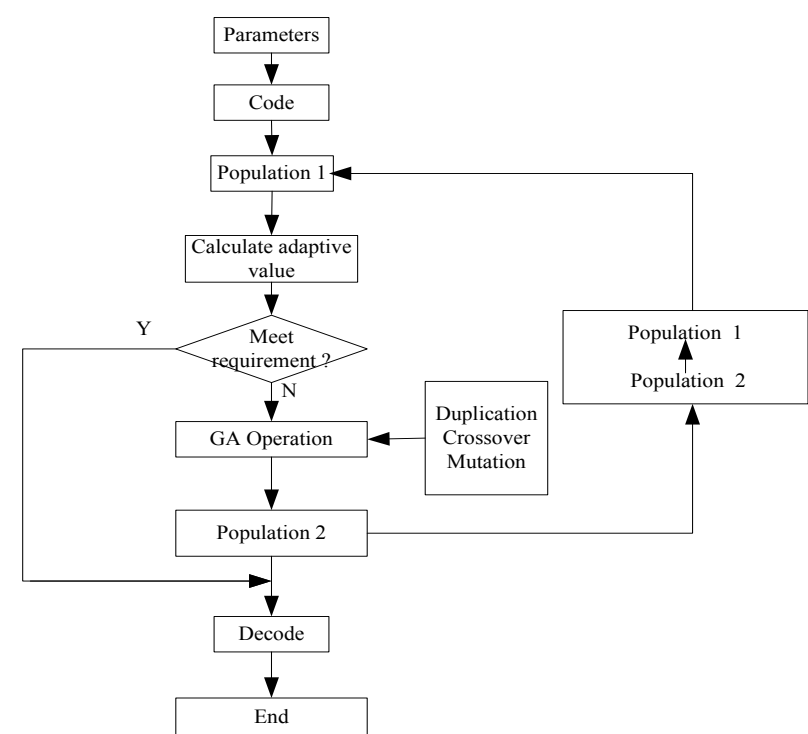

Fig. 3.the operation process of genetic algorithm .

\section{3 the tuning of PID parameters by genetic algorithm}

The PID parameter optimization of dynamic positioning system is to find suitable parameters to achieve the minimum value of $\mathbf{4 - 6 \mathbf { I }}$. For dynamic positioning ship three degrees of freedom, need nine parameters optimization, so many variables, if set a 
performance index function, affects the optimization precision of nine parameters, in order to solve this problem, after trying, we can independently optimization on three degrees of freedom, rather than the overall optimization, so we set the three performance index function index function matrix, this article selects a linear combination of the rise time and quantity of the deviation, control as the performance of the system control ${ }^{\mathbf{7}]}$ :

$J=\int_{0}^{t}\left(w_{1}|e(t)|+w_{2} \sqrt{|u(t)|}\right) d t+w_{3} t_{u}$

$J=\left[\begin{array}{l}J_{-} X \\ J_{-} Y \\ J_{-} \psi\end{array}\right]=\left[\begin{array}{l}\int_{0}^{t}\left(w_{1 x}\left|e_{x}(t)\right|+w_{2 x} \sqrt{\left|\tau_{X}\right|}\right) d t+w_{3 x} t_{x} \\ \int_{0}^{t}\left(w_{1 y}\left|e_{y}(t)\right|+w_{2 y} \sqrt{\left|\tau_{Y}\right|}\right) d t+w_{3 y} t_{y} \\ \int_{0}^{t}\left(w_{1 \psi}\left|e_{\psi}(t)\right|+w_{2 \psi} \sqrt{\left|\tau_{N}\right|}\right) d t+w_{3 \psi} t_{\psi}\end{array}\right]$

Based on the above analysis, the objective function will be taken from bottom, get fit function of genetic algorithm we need matrix, the genetic algorithm with adaptive function to evaluate group of PID parameters, in order to get the optimal PID parameters ${ }^{\text {[8-91 }}$

In this paper, the Matlab simulation is used to verify the overall flow chart of the design.

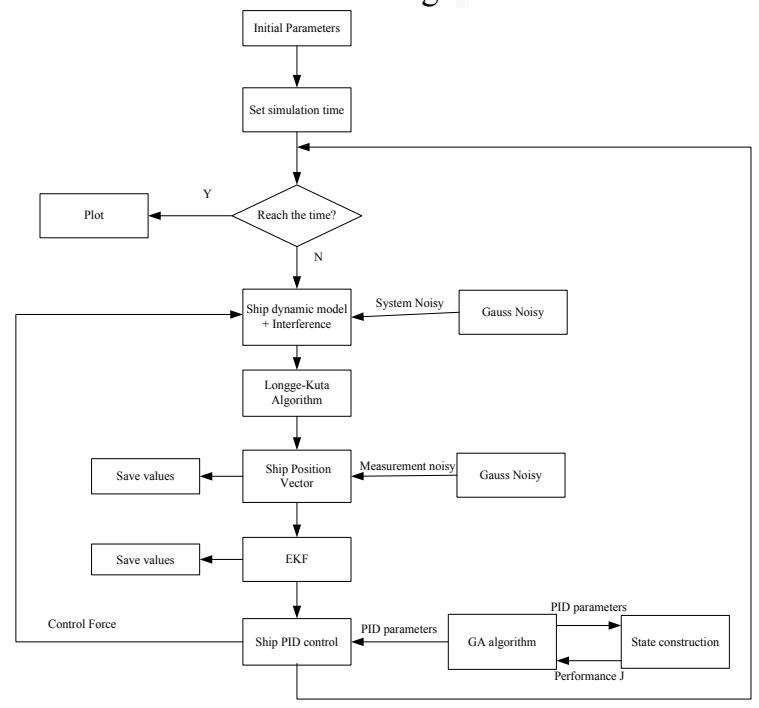

Fig. 4.system simulation flow chart .
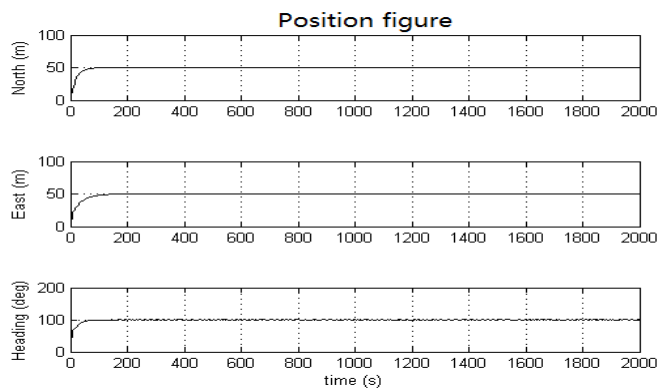
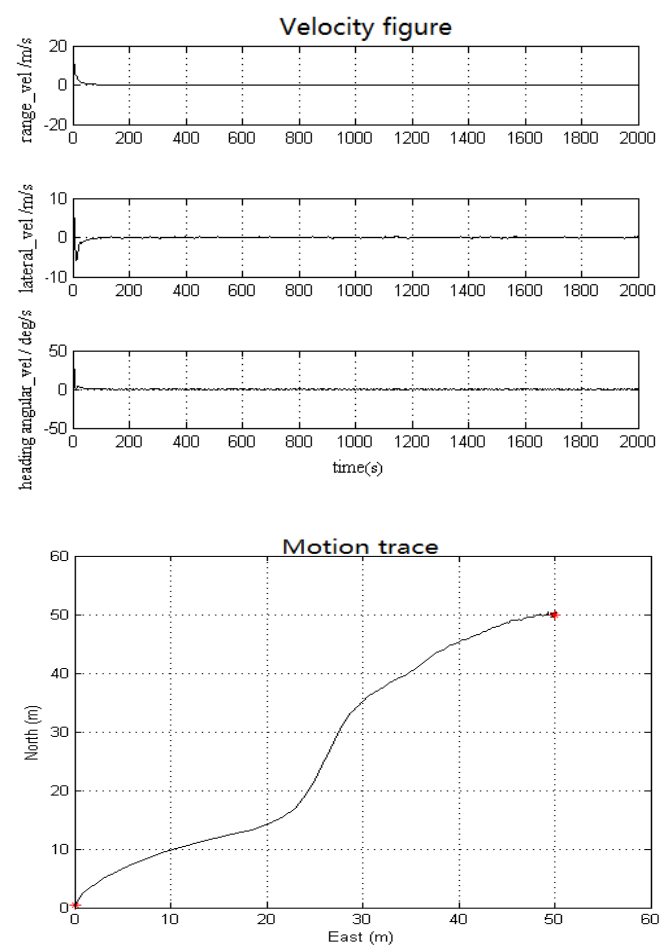

Fig. 5. control results of PID control parameters obtained by genetic algorithm

The change of performance indicator function and adaptation function in the optimization process of genetic algorithm is shown in figure6
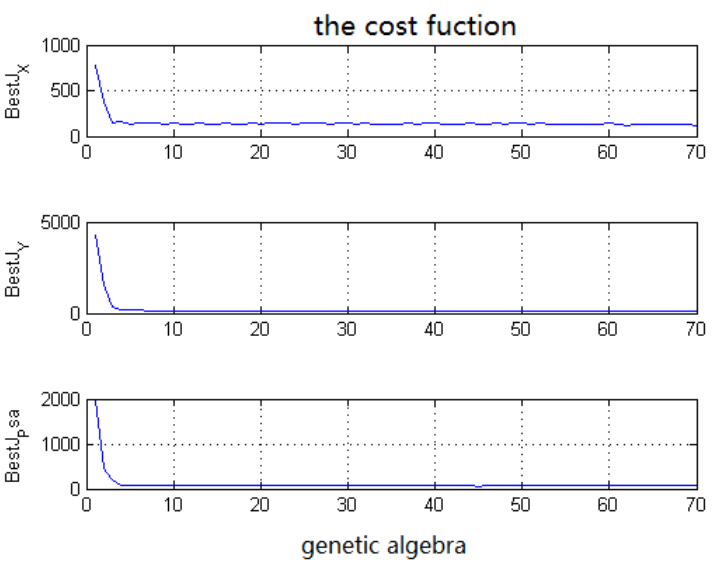

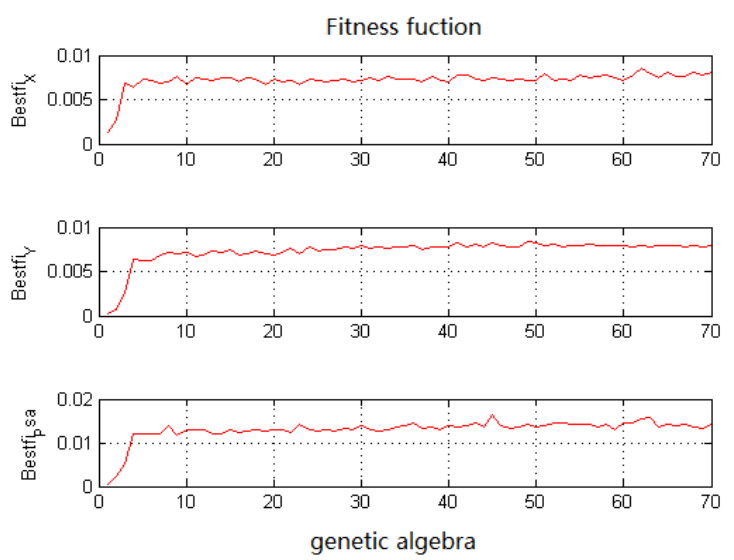

Fig. 6. shows the variation of performance indicator function and adaptation function in optimization of genetic algorithm

The control result of FIG. 5 shows that the PID parameters optimized by genetic algorithm achieve a good control effect. From FIG. 6, we can see that the convergence of genetic algorithm is very strong.

From the obtained effect picture, whether the location image or the speed image ${ }^{\mathbf{1} 10 \mathbf{1}}$, the optimized parameters are satisfied with the required accuracy.

In addition, through the simulation experiment for many times, found that the genetic algorithm in optimization of this case, there will be a good optimization results, I think it may be as intelligent algorithm, genetic algorithm itself will have certain randomness, is adopted in this paper can also be offline optimization, under the circumstance of state for refactoring may exist near the optimal solution under the local optimal value, have certain does not conform to the state of refactoring and the actual .

\section{4 conclusion}

From the simulation results, the PID parameter with genetic algorithm can also achieve the desired control effect. Gather together test through engineering personnel constantly experimental debugging, select a set of good group of PID parameters, gather together test relies heavily on the experience of the engineers, and will waste a lot of time and energy for engineers [9]. When the genetic algorithm is used to optimize the PID parameters, the simulation results show that the genetic algorithm can achieve the optimal control effect by optimizing the group of PID parameters. Thus, it is difficult to overcome the difficulty in adjusting PID parameters by engineering personnel.

\section{References}

1. boundary letter qian, fu mingyu, wang yuanhui. Ship dynamic positioning [M]. Beijing: science press, 2011:1-13.

2. jia xinle, Yang yansheng. Mathematical model of ship motion mathematics - mechanism modeling and identification modeling. Dalian: dalian maritime university press. 1999:294-359.
3. chung. Linear systems theory $[\mathrm{M}]$. Beijing: tsinghua university press, 2005.

4. A study on dynamic positioning of ships under mooring conditions [D]. Harbin: Harbin engineering university. 2013

5. Research on Marine dynamic positioning simulation technology [D] : China ship research institute; 2011.

6. Research on modeling and characteristics of ship movement [D] : wuhan university of technology; 2010.

7. Research on the dynamic positioning control of ships under single point mooring [D] : Harbin engineering university; 2013.

8. Math Works Inc. Genetic Algorithm and Direct Search Toolbox-MATLAB ${ }^{\circledR}$ version 2.4.1,User's guide, 2009.

9. Sefrioui M,Periaux J. Nash Genetic Algorithms: examples and applications[J].In:2000 Congress on Evolutionary Computation,volume1, IEEE Service Center,Piscataway, 2000-07:509 516.

10. Zhang Y-h, Jiang J-g, Jiang Y-j.Dynamic Positioning Nonlinear Control System of the Dredger Based on PROFINET IO[J].Journal of Computers,2013,8(2). 